(C) World Scientific Publishing Co. \& Operational Research Society of Singapore

DOI: $10.1142 / \mathrm{S} 0217595916990013$

\title{
Author Index \\ Volume 33
}

\section{Almomani, M. H. \& Alrefaei, M. H., Ordinal Optimization} with Computing Budget Allocation for Selecting an Optimal Subset

2 (2016) 1650009

Alrefaei, M. H., see Almomani, M. H.

2 (2016) 1650009

Avinadav, T., Stochastic Periodic-Review Models with Duration- and Quantity-Dependent Inventory Costs:

Properties and Approximations

4 (2016) 1650030

Azizi, R. \& Matin, R. K., Ranking Two-Stage Production Units in Data Envelopment Analysis

1 (2016) 1650002

Babaie-Kafaki, S. \& Ghanbari, R., Descent Symmetrization of the Dai-Liao Conjugate Gradient Method

Baek, J., see Park, J. H.

2 (2016) 1650008

4 (2016) 1650024

5 (2016) 1650036

Bernard, C. \& McLeish, D., Algorithms for Finding Copulas Minimizing Convex Functions of Sums

Celik, N., see Thanos, A. E.

Celik, N., see $\mathrm{Xu}, \mathrm{J}$.

(Victor) Chan, W. K., Linear Programming Formulation of Idle Times for Single-Server Discrete-Event Simulation Models

5 (2016) 1650040

1 (2016) 1650006

3 (2016) 1650017

Chen, C.-H., see Xu, J.

Chen, D.-R., see Cui, L.

Chen, K., Xu, R. \& Fang, H., Information Disclosure Model Under Supply Chain Competition with Asymmetric Demand Disruption

Chen, T.-Y., see Chung, L.-Y.

6 (2016) 1650043

6 (2016) 1650052

Chen, X., see Meng, F.

Chew, E. P., see Tao, Y.

1 (2016) 1650007

4 (2016) 1650029

Choi, B.-C. \& Park, M.-J., An Ordered Flow Shop with Two Agents

5 (2016) 1650037

Chopra, R., see Kumar, S.

6 (2016) 1650047 
Chung, L.-Y., Liao, Y.-H., Hsieh, W.-Y., Chen, T.-Y. \& Lin, C.-K., Alternative Reductions and Axiomatizations of the Unit-Level-Core

6 (2016) 1650052

Cui, L., Liu, L., Chen, D.-R. \& Xie, J.-F., Recovery of Low Rank Symmetric Matrices via Schatten p Norm

Minimization

1 (2016) 1650003

6 (2016) 1650044

Dai, Q., see Wu, D.

Datta, S., see Sen, D. K.

Deng, Z., see Luo, J.

Du, J., Huo, J. \& Zhu, J., Data Envelopment Analysis with Output-Bounded Data

5 (2016) 1650033

6 (2016) 1650046

6 (2016) 1650050

El-Hadidy, M. A., On Maximum Discounted Effort Reward Search Problem

3 (2016) 1650019

Ensor, A. \& Lillo, F., Colored-Edge Graph Approach for the Modeling of Multimodal Transportation Systems

1 (2016) 1650005

Eydi, A. \& Fazli, L., A Multi-Period Multiple Objective

Uncertain Programming Model to Allocate Order for

Supplier Selection Problem

Fan, P., see Shi, Y.

Fang, H., see Chen, K.

Fang, S.-C., see Luo, J.

Fazli, L., see Eydi, A.

Feng, X.-Q., see Tan, Q.-Y.

Ghanbari, R., see Babaie-Kafaki, S.

Goswami, V., see Panda, G.

Govindan, K., see Manik, P.

Guo, X., see Luo, J.

Gupta, A., see Manik, P.

Hewitt, M., Nowak, M. \& Nataraj, N., Planning Strategies for Home Health Care Delivery

Hsieh, W.-Y., see Chung, L.-Y.

Huang, E., see $\mathrm{Xu}$, J.

Huang, H., see $\mathrm{Xu}$, B.

Huo, J., see Du, J.

Jeong, B. H., see Zhang, Y.

Jha, P. C., see Manik, P.

Ji, X., see Ma, W.

Jung, J., see Park, J. H.

Kazemi Matin, R., see Mirjaberi, M.

Kumar, S., Chopra, R. \& Saxena, R. R., A Fast Approach to

Solve Matrix Games with Payoffs of Trapezoidal Fuzzy

Numbers

6 (2016) 1650047

Lee, L. H., see Tao, Y.

4 (2016) 1650029 
Lee, L. H., see Xu, J.

Li, C.-L. \& Li, Q., Polynomial-Time Solvability of Dynamic Lot Size Problems

Li, J., Wang, Q., Yan, H. \& Zhu, S. X., Optimal

Remanufacturing and Pricing Strategies Under

Name-Your-Own-Price Auctions and Stochastic Demand

$\mathrm{Li}$, J., see $\mathrm{Wu}, \mathrm{D}$.

Li, Q., see Li, C.-L.

Li, X., see Shi, Y.

Lian, Z., see Shi, Y.

Liao, Y.-H., see Chung, L.-Y.

Lillo, F., see Ensor, A.

Lin, C.-K., see Chung, L.-Y.

Liu, C., Xiang, X., Zhang, C. \& Zheng, L., A Decision

Model for Berth Allocation Under Uncertainty Considering

Service Level Using an Adaptive Differential Evolution

Algorithm

Liu, C.-L. \& Wang, J.-J., Unrelated Parallel-Machine

Scheduling with Controllable Processing Times and Impact of Deteriorating Maintenance Activities under

Consideration

Liu, L., see Cui, L.

Liu, Q., see Tan, Q.-Y.

Liu, S.-C. \& Wu, C.-C., A Faster FPTAS for a Supply Chain Scheduling Problem to Minimize Holding Costs with Outsourcing

Liu, Y., On Low Rank Approximation of Linear Operators in $p$-Norms and Some Algorithms

$\mathrm{Lu}, \mathrm{Y}$. , see $\mathrm{Wu}, \mathrm{J}$.

Lu, Y.-Y., see Wang, Z.

Luo, J., Fang, S.-C., Deng, Z. \& Guo, X., Soft Quadratic Surface Support Vector Machine for Binary Classification

Ma, W. \& Ji, X., Online Work-Break Problem and its Competitive Analysis

$\mathrm{Ma}, \mathrm{W}$. , see $\mathrm{Xu}, \mathrm{B}$.

Mahapatra, S. S., see Sen, D. K.

Manik, P., Gupta, A., Jha, P. C. \& Govindan, K., A Goal Programming Model for Selection and Scheduling of Advertisements on Online News Media

Matin, R. K., see Azizi, R.

McLeish, D., see Bernard, C.
3 (2016) 1650017

3 (2016) 1650018

1 (2016) 1650004

6 (2016) 1650044

3 (2016) 1650018

6 (2016) 1650051

3 (2016) 1650022

6 (2016) 1650052

1 (2016) 1650005

6 (2016) 1650052

6 (2016) 1650049

1 (2016) 1650001

1 (2016) 1650003

5 (2016) 1650035

5 (2016) 1650039

4 (2016) 1650023

4 (2016) 1650025

4 (2016) 1650032

6 (2016) 1650046

2 (2016) 1650011

4 (2016) 1650028

5 (2016) 1650033

2 (2016) 1650012

1 (2016) 1650002

5 (2016) 1650040 
Meng, F. \& Chen, X., Cooperative Fuzzy Games with Convex Combination Form

1 (2016) 1650007

Mirjaberi, M. \& Kazemi Matin, R., On the Calculation of Directional Scale Elasticity in Data Envelopment Analysis

4 (2016) 1650026

Mostafaee, A. \& Soleimani-Damaneh, M., Some Conditions for Characterizing Anchor Points

Nataraj, N., see Hewitt, M.

Nowak, M., see Hewitt, M.

Panda, G., Goswami, V. \& Banik, A. D., Equilibrium and Socially Optimal Balking Strategies in Markovian Queues with Vacations and Sequential Abandonment

Park, J. H., Jung, J. \& Baek, J., Modeling and Analysis of Zone-Based Registration in Mobile Communication Network by Considering Busy-Line Effect and Implicit Registration

4 (2016) 1650024

5 (2016) 1650037

Park, M.-J., see Choi, B.-C.

Rachakonda, S. R., see Rallabandi, L. N. P. K.

3 (2016) 1650020

Rallabandi, L. N. P. K., Vandrangi, R. \& Rachakonda, S. R., Improved Consistency Ratio for Pairwise Comparison Matrix in Analytic Hierarchy Processes

Razavyan, S., A Method for Generating a Well-Distributed Pareto Set in Multiple Objective Mixed Integer Linear Programs Based on the Decision Maker's Initial Aspiration Level

2 (2016) 1650013

5 (2016) 1650041

5 (2016) 1650041

5 (2016) 1650036

Sáenz, J. P., see Thanos, A. E.

Saxena, R. R., see Kumar, S.

Sedeño-Noda, A., Ranking One Million Simple Paths in Road Networks

3 (2016) 1650020

Sen, D. K., Datta, S. \& Mahapatra, S. S., A TODIM-Based Decision Support Framework for G-Resilient Supplier Selection in Fuzzy Environment

5 (2016) 1650033

Shi, Y. \& Lian, Z., Equilibrium Strategies and Optimal Control for a Double-Ended Queue

Shi, Y., Li, X. \& Fan, P., Optimization of an $M / M / \infty$ Queueing System with Free Experience Service

Soleimani-Damaneh, M., see Mostafaee, A.

4 (2016) 1650031

1 (2016) 1650006

6 (2016) 1650047

5 (2016) 1650042

Tan, Q.-Y., Wei, C.-P., Liu, Q. \& Feng, X.-Q., The Hesitant Fuzzy Linguistic TOPSIS Method Based on Novel

Information Measures

Tang, H., see Zhao, C.

5 (2016) 1650035

6 (2016) 1650048

Tao, Y., Lee, L. H. \& Chew, E. P., Quantifying the Effect of Sharing Information in a Supply Chain Facing Supply Disruptions 
Thanos, A. E., Celik, N. \& Sáenz, J. P., An Evolutionary

Sequential Sampling Algorithm for Multi-Objective

Optimization

Vandrangi, R., see Rallabandi, L. N. P. K.

Waligóra, G., Comparative Analysis of Some Metaheuristics for Discrete-Continuous Project Scheduling with Activities of Identical Processing Rates

Wang, J.-J., see Liu, C.-L.

Wang, Q., see Li, J.

Wang, Z., Wei, C.-M. \& Lu, Y.-Y., Permutation Flow Shop Problem with Shortening Job Processing Times

Wang, Z., Wei, C.-M. \& Wu, Y.-B., Single Machine

Two-Agent Scheduling with Deteriorating Jobs

Wei, C.-M., see Wang, Z.

Wei, C.-M., see Wang, Z.

Wei, C.-P., see Tan, Q.-Y.

Wirth, A., see Zhang, L.

Won, Y., Dominance Relationship Among the Retailer's

Strategies Under the Semi-Stackelberg Newsvendor

Situation with Quantity Discounts

Wu, C.-C., see Liu, S.-C.

Wu, D., Xie, Y., Dai, Q. \& Li, J., A Systematic Overview of Operations Research/Management Science Research in Mainland China: Bibliometric Analysis of the Period 2001-2013

Wu, J., Zhang, Y., Zhang, L. \& Lu, Y., A Sequential Convex Program Approach to an Inverse Linear Semidefinite

Programming Problem

Wu, Y.-B., see Wang, Z.

Xiang, X., see Liu, C.

Xie, J.-F., see Cui, L.

Xie, Y., see Wu, D.

Xu, B., Ma, W., Huang, H. \& Yue, L., Weighted Constrained Position Shift Model for Aircraft Arrival Sequencing and Scheduling Problem

Xu, J., Zhang, S., Huang, E., Chen, C.-H., Lee, L. H. \& Celik, N., MO $O^{2}$ TOS: Multi-Fidelity Optimization with Ordinal Transformation and Optimal Sampling

$\mathrm{Xu}, \mathrm{R}$., see Chen, K.

Yan, H., see Li, J.

Yuan, X. G. \& Zhu, N., Bullwhip Effect Analysis in

Two-Level Supply Chain Distribution Network Using

Different Demand Forecasting Technology
1 (2016) 1650006

3 (2016) 1650020

3 (2016) 1650015

1 (2016) 1650001

1 (2016) 1650004

4 (2016) 1650032

5 (2016) 1650034

4 (2016) 1650032

5 (2016) 1650034

5 (2016) 1650035

4 (2016) 1650027

2 (2016) 1650014

5 (2016) 1650039

6 (2016) 1650044

4 (2016) 1650025

5 (2016) 1650034

6 (2016) 1650049

1 (2016) 1650003

6 (2016) 1650044

4 (2016) 1650028

3 (2016) 1650017

6 (2016) 1650043

1 (2016) 1650004

3 (2016) 1650016 
Yue, L., see Xu, B.

Zhang, C., see Liu, C.

Zhang, L. \& Wirth, A., Online Machine Scheduling with Family Setups

Zhang, L., see Wu, J.

Zhang, L., see Zhang, N.

Zhang, N. \& Zhang, L., No Gap Second-order Optimality Conditions for a Matrix Cone Programming Induced by the Nuclear Norm

Zhang, S., see Xu, J.

Zhang, Y. \& Jeong, B. H., Development of a Multi-period Output Model for Considering Time Lag Effect

Zhang, Y., see Wu, J.

Zhao, C. \& Tang, H., Scheduling Deteriorating Jobs with Availability Constraints to Minimize the Makespan

Zheng, L., see Liu, C.

Zhu, J., see Du, J.

Zhu, N., see Yuan, X. G.

Zhu, S. X., see Li, J.
4 (2016) 1650028

6 (2016) 1650049

4 (2016) 1650027

4 (2016) 1650025

2 (2016) 1650010

2 (2016) 1650010

3 (2016) 1650017

3 (2016) 1650021

4 (2016) 1650025

6 (2016) 1650048

6 (2016) 1650049

6 (2016) 1650050

3 (2016) 1650016

1 (2016) 1650004 